\title{
EPIFITISMO VASCULAR EM UMA FLORESTA DE RESTINGA DO BRASIL SUBTROPICAL
}

Jorge Luiz Waechter

Departamento de Botânica, IB

UFRGS, Porto Alegre, RS

\section{RESUMO}

Um estudo fitossociológico de epífitos vasculares foi realizado em uma floresta de restinga do nordeste do Rio Grande do Sul, Brasil. Um total de 60 árvores hospedeiras (forófitos) foi amostrado empregando o método dos quadrantes centrados, incluindo árvores com no mínimo $10 \mathrm{~cm}$ de diâmetro à altura do peito. Os epífitos vasculares registrados pertencem a 8 famílias, 20 gêneros e 31 espécies, sendo as Orchidaceae e as Bromeliaceae as mais diversificadas. Diversas espécies adicionais foram registradas no estande florestal de aproximadamente um hectare. Valores de freqüência dos epífitos vasculares formaram uma seqüência gradualmente decrescente de espécies, sendo Microgramma vacciniifolia (Polypodiaceae) e Tillandsia aëranthos (Bromeliaceae) as mais importantes. O índice de diversidade de Shannon foi estimado ( $\left.H^{\prime}=2,990\right)$, usando a freqüência sobre forófitos individuais como uma medida de participação quantitativa (pi). O número de epífitos vasculares sobre forófitos individuais variou de 4 a 16 espécies, apresentando uma distribuição polimodal e um valor modal mais alto de oito espécies.

Palavras chaves: epífitos vasculares, mata de restinga, fitossociologia, Sul do Brasil. 


\section{ABSTRACT}

A phytosociological survey of vascular epiphytes was performed in a sand-dune forest (restinga) of northeastern Rio Grande do Sul, Southern Brazil. A total of 60 host trees (phorophytes) were sampled according to the point-centered quarter method, including trees with at least $10 \mathrm{~cm}$ of diameter at breast height. The recorded vascular epiphytes belong to 8 families, 20 genera and 31 species, the Orchidaceae and Bromeliaceae being the most diversified. Several additional species were recorded in the whole forest stand with ca. one hectare. Frequency values of vascular epiphytes formed a gradually decreasing sequence of species, the most important being Microgramma vacciniifolia (Polypodiaceae) and Tillandsia aëranthos (Bromeliaceae). The Shannon index of diversity was estimated ( $\mathrm{H}^{\prime}$ $=2,990$ ), using frequency on individual phorophytes as a measure of quantitative participation (pi). The number of vascular epiphytes on individual phorophytes ranged from 4 to 16 species, showing a polymodal distribution and a highest modal value of 8 species.

Key words: vascular epiphytes, sand-dune forest, phytosociology, Southern Brazil.

\section{INTRODUÇÃO}

Os epífitos vasculares constituem um dos tipos biológicos mais característicos de florestas tropicais e subtropicais úmidas, tendo merecido capítulos detalhados na maioria das publicações que analisam e descrevem estas formações (Richards, 1952; Walter, 1971; Lüttge, 1997). As temperaturas mais ou menos elevadas, associadas a precipitações intensas e regulares, podem favorecer o desenvolvimento de comunidades epifíticas altamente diversificadas, cuja composição florística e estrutura comunitária ainda são pouco conhecidas.

A região neotropical apresenta uma das floras epifíticas mais 
diversificadas do mundo. Aspectos taxonômicos, ecológicos e geográficos desta flora foram discutidos pioneiramente por Schimper (1888) e, mais recentemente, por Gentry \& Dodson (1987) e Benzing (1989). Nas últimas décadas, um número considerável de trabalhos tem sido direcionado ao conhecimento da composição florística e da estrutura sinusial do componente epifítico vascular de diferentes formações, desde florestas chuvosas ou alagadas de terras baixas (Valdivia, 1977; Kelly, 1985; Waechter, 1986; ter Steege \& Cornelissen, 1989) até florestas sazonais ou nebulares de terras altas (Sugden \& Robins, 1979; Nadkarni, 1985; Cattling \& Lefkovitch, 1989; Brown, 1990).

A alta diversidade taxonômica e as dificuldades de acesso ao dossel provavelmente determinaram um outro tipo de enfoque nos estudos com epífitos vasculares, ou seja, o direcionamento a uma determinada categoria taxonômica. Neste sentido, destacam-se os trabalhos realizados com pteridófitos de modo geral (Sota, 1971, 1972; Evangelista, 1996; Senna \& Waechter, 1997) ou com as duas famílias mais diversificadas no dossel de florestas neotropicais, Bromeliaceae (Pittendrigh, 1948;Veloso, 1952; Veloso \& Calabria, 1953; Sugden, 1981; Fontoura, 1995) e Orchidaceae (Braga, 1977; Migenis \& Ackerman, 1993; Waechter, 1998; Nunes \& Waechter, 1998).

A caracterização florística e estrutural das matas de restinga, bem como de outras formações arbustivas e herbáceas relacionadas a depósitos costeiros quaternários, tem sido discutida por diversos autores em numerosas publicações (Ule, 1901; Hueck, 1955; Henriques et al., 1986; Araújo, 1987, 1992). As matas de restinga do Rio Grande do Sul foram descritas pioneiramente por Lindman (1906) e Malme (1936). Mais recentemente, Waechter $(1985,1990)$ revisou as principais tipologias vegetacionais encontradas na extensa planície costeira sul-rio-grandense, distinguindo três tipos principais de florestas: matas arenosas, matas turfosas e matas ciliares (galerias).

A maioria dos estudos realizados em florestas de restingas destaca, de maneira mais ou menos detalhada, a importância florística ou 
fisionômica do componente epifítico vascular (Hertel, 1959; Reitz, 1961; Baptista et al., 1979; Lindeman et al., 1975; Waechter, 1985, 1990). Para o litoral do Rio Grande do Sul, um número relativamente grande de espécies epifíticas foi citado por Rambo (1954, 1961), que chama a atenção para a origem tropical e a diluição latitudinal desta categoria ecológica. Posteriormente Waechter $(1992,1998)$ documentou o gradiente decrescente de riqueza epifítica vascular, no sentido norte-sul da planície costeira.

O objetivo do presente trabalho é estudar a composição taxonômica, a participação quantitativa e a diversidade específica de epífitos vasculares em uma floresta de restinga do Rio Grande do Sul.

\section{ÁREA DE ESTUDO}

O remanescente florestal estudado situa-se na localidade de Emboaba ( $29^{\circ} 58^{\prime} \mathrm{S}$ e $\left.50^{\circ} 14^{\prime} \mathrm{W}\right)$, no município de Osório, no litoral norte do Rio Grande do Sul, a uma distância de $10 \mathrm{~km}$ do Oceano Atlântico (Figura 1). O relevo da área, assim como praticamente de toda a planície costeira interna, apresenta-se plano a suavemente ondulado, com altitudes oscilando em torno de $20 \mathrm{~m}$ sobre o nível do mar.

O clima da região, como em todo litoral sul-rio-grandense, é subtropical úmido, sendo classificado no tipo Cfa do sistema de Köppen (Mota, 1951; Moreno, 1961). O diagrama climático, elaborado conforme Walter (1986) para a Estação Meteorológica de Imbé (Figura 2), uma das mais próximas do local, registra médias anuais de temperatura e pluviosidade iguais a $19,2^{\circ} \mathrm{C}$ e $1325,6 \mathrm{~mm}$, respectivamente (dados fornecidos pelo DEPRC - Departamento Estadual de Portos, Rios e Canais). As chuvas diminuem sensivelmente nos meses de maio e novembro (primavera e outono), sem contudo determinar uma estação seca. As temperaturas mensais decrescem acentuadamente nos meses de inverno, porém o número médio de geadas anuais é relativamente baixo, inferior a 5 dias (Nimer, 1979). Os ventos predominantes de nordeste, aliados à maresia, 
freqüentemente imprimem feições unilaterais às copas de árvores isoladas (Waechter, 1985, 1990).

O solo sobre o qual se desenvolve a mata de restinga de Emboaba pertence à Unidade de Mapeamento Osório, classificada como Areias Quartzosas Distróficas (Haplumbrepts), derivadas de sedimentos não consolidados do Quaternário recente (Lemos et al., 1973). As principais características físicas destes solos são a grande profundidade (até mais de $3 \mathrm{~m}$ ), a coloração bruna ou amarelada, a textura arenosa e a conseqüente baixa capacidade de retenção de água. Sob o ponto de vista químico, os solos são fortemente ácidos, com baixo conteúdo de matéria orgânica e baixa fertilidade natural. A remoção da vegetação natural geralmente causa problemas de erosão e eluviação.

Um estudo fitossociológico do componente arbóreo, realizado no mesmo estande florestal (Dillenburg et al., 1992), evidenciou a ocorrência de 15 espécies arbóreas, formando um estrato arbóreo único, com um dossel irregular de aproximadamente sete metros de altura. Neste estrato constatou-se, segundo diversos parâmetros populacionais, a dominância de Sebastiania commersoniana (Euphorbiaceae). O índice de diversidade arbórea $\left(H^{\prime}=1,975\right)$, incluindo árvores com DAP mínimo de $5 \mathrm{~cm}$, pode ser considerado baixo.

\section{MATERIAL E MÉTODOS}

As árvores hospedeiras (forófitos), consideradas como unidades amostrais, foram levantadas a partir do método dos quadrantes centrados (Cottan \& Curtis,1956; Mueller-Dombois \& Ellenberg, 1974). Em um estande florestal de aproximadamente um hectare, foram demarcados 100 pontos de amostragem, dispostos de maneira regular, com uma distância de $10 \mathrm{~m}$ entre si. Com o auxílio de uma seqüência de números aleatórios, foram definidos 15 pontos de amostragem, que resultaram em 60 árvores hospedeiras. Os quadrantes foram estabelecidos por meio de uma 
cruzeta giratória, instalada no ápice de estacas correspondentes aos pontos de amostragem.

O diâmetro mínimo considerado para inclusão das árvores foi de $10 \mathrm{~cm}$, medidos à altura do peito $(1,30 \mathrm{~m})$. Quando a altura do fuste era inferior a este limite, amostravam-se as árvores que apresentassem pelo menos um ramo com diâmetro igual ou superior a $10 \mathrm{~cm}$. A altura total das árvores foi calculada pelo método trigonométrico, utilizando-se um clinômetro para obter o ângulo entre a horizontal e o ápice da árvore, e uma trena, para medir a distância entre o observador e o fuste da árvore.

As espécies epifíticas vasculares foram registradas pela observação macroscópica direta, quase sempre acompanhada pela escalada da árvore amostrada. Em muitos casos foi também empregada a observação telescópica, utilizando um binóculo de alta resolução óptica. A identificação das espécies foi baseada em floras regionais e revisões taxonômicas recentes. Exemplares coletados na área de estudo foram depositados no Herbário do Departamento de Botânica da Universidade Federal do Rio Grande do Sul (ICN).

A representatividade florística do levantamento fitossociológico foi avaliada pela relação cumulativa entre número de unidades amostrais e número de espécies epifíticas (curva de coletor), considerando sucessivamente as quatro árvores hospedeiras correspondentes a cada ponto de amostragem. A participação quantitativa das espécies epifíticas foi estimada pelas freqüências absoluta e relativa sobre forófitos individuais (FAi, FRi) e forófitos específicos (FAj, FRj). Um valor de importância epifítica (Vle) foi estimado a partir dos dois tipos de freqüência relativa. As fórmulas utilizadas foram:

$\mathrm{FAi}=(\mathrm{Nfi} / \mathrm{Nfa}) .100$

$\mathrm{FRi}=(\mathrm{Nfi} / \Sigma \mathrm{Nfi}) \cdot 100$

$\mathrm{FAj}=(\mathrm{Sfi} / \mathrm{Sfa}) .100$

$\mathrm{FRj}=(\mathrm{Sfi} / \Sigma \mathrm{Sfi}) \cdot 100$

$V l e=(F R i+F R j) / 2$ 
onde,

$\mathrm{Nfi}$ = número de indivíduos forofíticos ocupados pela espécie $i$ epifítica

$\mathrm{Nfa}=$ número total de indivíduos forofíticos amostrados

$\mathrm{Sfi}=$ número de espécies forofíticas ocupadas pela espécie $i$ epifítica

Sfa $=$ número total de espécies forofíticas amostradas

Vle $=$ valor de importância das espécies epifíticas

A diversidade da sinúsia epifítica foi estimada pelo índice de Shannon (Whittaker, 1972; Magurran, 1988), utilizando a fórmula H' = - $\Sigma$ pi . $\log \mathrm{pi}$, considerando como probabilidade participativa de cada espécie (pi) a freqüência absoluta decimal sobre forófitos individuais.

\section{RESULTADOS E DISCUSSÃO}

O levantamento fitossociológico de epífitos vasculares resultou em 31 espécies, pertencendo a 20 gêneros e 8 famílias (Tabela 1). As famílias mais ricas em espécies foram Orchidaceae (12) e Bromeliaceae (8), seguidas à distância por Piperaceae (3), Polypodiaceae (3), Cactaceae (2) e demais famílias com apenas uma espécie. Estas mesmas famílias encontram-se também entre as mais ricas em diversas outras florestas neotropicais (Valdivia, 1977; Nadkarni, 1985; Gentry \& Dodson, 1987). Na Planície Costeira do Rio Grande do Sul, as florestas brejosas ou turfosas apresentam um epifitismo vascular mais diversificado (Waechter, 1986; 1992).

Um total de 22 espécies adicionais foram observadas e registradas no remanescente florestal, resultando em uma flora epifítica total de 12 famílias, 32 gêneros e 53 espécies (Tabela 1). As famílias não amostradas ou não incluídas no levantamento fitossociológico foram: Cecropiaceae e Moraceae, epifíticas apenas no estádio inicial, tornando-se na idade adulta árvores grandes, importantes na fisionomia das restingas; 
Ophioglossaceae e Vittariaceae, samambaias pendentes raras na área de estudo, observadas apenas entre as bainhas persistentes de Butia capitata (butiá-da-praia). A maior proporção de espécies raras (não amostradas) foi verificada em Polypodiaceae.

Estudos realizados em ambientes costeiros do hemisfério norte indicam um epifitismo vascular menos diversificado, possivelmente influenciado pelo clima sazonal ou pela estrutura da vegetação. Na região tropical atlântica do México, um matorral costeiro apresentou apenas 6 espécies de Tillandsia (Bromeliaceae), um gênero altamente xerofítico (Garcia-Franco, 1996), enquanto uma floresta alagada evidenciou 5 famílias e 15 espécies (Zimmerman \& Olmsted, 1992). No sul da Flórida (EUA), já em latitudes subtropicais, 3 famílias e 18 espécies de epífitos vasculares foram amostrados por Bennett (1986): Orchidaceae (9), Bromeliaceae (8) e Piperaceae (1).

Um dos aspectos interessantes do epifitismo de Emboaba foi a observação de espécies predominantemente terrestres no ambiente epifítico (Bromelia antiacantha, Cereus hildmannianus, Rumohra adiantiformis, Tradescantia fluminensis) e de espécies predominantemente epifíticas no ambiente terrestre (Aechmea recurvata, Peperomia caulibarbis, Peperomia pereskiifolia e Polypodium catharinae). Estas observações coincidem com a classificação de categorias ecológicas baseadas na relação com as árvores hospedeiras (Benzing, 1990). Aspectos ambientais, como o clima úmido e o solo arenoso bem drenado, além de aspectos estruturais das matas de restinga, como o porte baixo e a existência de clareiras, podem favorecer esta ocupação ampla de substratos disponíveis.

Os 60 forófitos amostrados, relacionados aos 15 pontos de amostragem, pertenceram a 12 espécies diferentes (Tabela 2), com uma evidente dominância de Sebastiania commersoniana (branquilho), conforme já constatado no estudo anterior de Dillenburg et al. (1992). Outras árvores que se destacaram pelo número de indivíduos foram Casearia sylvestris (chá-de-bugre) e Myrsine umbellata (capororoca). As árvores amostradas 
apresentaram alturas entre 5 e 12,5m (média $=6,7 \mathrm{~m}$ ) e diâmetros entre $10 \mathrm{e}$ $90 \mathrm{~cm}$ (média $=19,8 \mathrm{~cm}$ ). A maior árvore amostrada foi um indivíduo de Sideroxylon obtusifolium (espinheira, coronilha), cujas dimensões excêntricas levam a crer que a estrutura atual da floresta deve resultar de interferências antrópicas.

A relação cumulativa entre número de pontos de amostragem e número de espécies epifíticas, assim como de espécies arbóreas (Figura 3) mostra que o levantamento foi numericamente representativo. A partir de aproximadamente cinco pontos quadrantes (20 árvores amostradas), não se verifica mais um aumento acentuado no número de espécies epifíticas. A relação evidencia também que a riqueza específica do componente epifítico supera amplamente a do componente arbóreo.

O número de espécies epifíticas por forófito individual variou de 4 a 16, apresentando uma distribuição polimodal e um valor modal mais alto correspondente a 8 espécies (Figura 4). Todas as árvores amostradas, portanto, apresentaram pelo menos algumas espécies epifíticas, mesmo aquelas com aspecto arquitetônico altamente diferenciado no contexto geral, como Butia capitata (butiá-da-praia) e Cereus hildmannianus (tuna, mandacaru).

O número máximo de 16 espécies epifíticas foi registrado sobre três forófitos diferentes (Tabela 2, Figura 4), pertencendo às espécies Sebastiania commersoniana (branquilho), Chrysophyllum marginatum (aguaívermelho) e Eugenia uniflora (pitangueira). De modo geral, não se verificou correlação entre altura total ou diâmetro à altura do peito dos forófitos e riqueza epifítica vascular. Uma porcentagem relativamente alta de árvores $(45,0 \%)$ apresentou fustes inferiores a $1,30 \mathrm{~m}$, ou seja, com dois a cinco ramos à altura do peito. Este aspecto pode favorecer o epifitismo sobre árvores pequenas, pelo aumento de substrato disponível.

Em regiões tropicais úmidas, o número máximo de espécies epifíticas por forófito individual pode exceder amplamente o valor registrado neste estudo (Sanford, 1968; Johansson, 1974; Vareschi, 1980). Em dois estandes de florestas costeiras turfosas, em latitudes diferentes da Planície 
Costeira do Rio Grande do Sul, foram registrados números máximos de 19 e 35 espécies epifíticas vasculares, sobre exemplares de Erythrina crista-galli (corticeira) e Ficus organensis (figueira), respectivamente (Waechter, 1992).

As espécies epifíticas vasculares apresentaram uma seqüência decrescente mais ou menos gradual na floresta estudada, considerando os valores de freqüência sobre indivíduos e espécies de forófitos amostrados (Tabela 3, Figura 5). Considerando o valor de importância ( $\mathrm{Vle}$ ), derivado das estimativas de freqüência, duas espécies destacaram-se como dominantes na seqüência: Microgramma vaccinifolia (Polypodiaceae) e Tillandsia aëranthos (Bromeliaceae). Apesar das diferenças taxonômicas e ecológicas (hábito reptante versus rosetado), as duas espécies coincidem quanto à síndrome de dispersão anemófila, respectivamente através de esporos diminutos e sementes plumosas.

Considerando as dez espécies epifíticas mais importantes, cinco foram Bromeliaceae, ocupando todos os postos subseqüentes ao primeiro (Tabela 3). Durante o trabalho de campo, foi constatada a importância desta família para a fisionomia geral da floresta. A abundância de Tillandsia usneoides confere uma tonalidade acinzentada à restinga de Emboaba, enquanto Aechmea recurvata, durante a floração no final do inverno e início da primavera, tinge a mata com a coloração vermelhobrilhante das folhas involucrais. O número de espécies raras (freqüência absoluta inferior a $5 \%$ ) foi relativamente pequeno, quando se considera levantamentos fitossociológicos realizados em regiões tropicais e subtropicais úmidas.

Comparando os valores de freqüência sobre indivíduos e espécies de forófitos (Tabela 3, Figura 5), verifica-se uma correlação positiva, ou seja, quanto maior a abundância na floresta, maior o número de substratos diferentes ocupados, considerando cada espécie arbórea como traduzindo um padrão básico de ramificação, ritidoma e folhagem. A relação mostra também, como já amplamente conhecido, que a maioria das espécies epifíticas não apresenta especificidade ou grau de restrição quanto a uma 
determinada espécie de árvore hospedeira (Benzing, 1990).

$\mathrm{O}$ índice de diversidade epifítica $\left(\mathrm{H}^{\prime}\right)$ na restinga de Emboaba foi estimado em 2,990, um valor que pode ser considerado como relativamente baixo. As causas possiveis para esta baixa diversidade podem ser a baixa retenção hídrica e a escassez de nutrientes minerais nos solos de restingas arenosas (Dillenburg et al., 1992), que determinam florestas com características xeromórficas e, segundo Gentry \& Dodson (1987), um epifitismo vascular menos diversificado. Um certo grau de interferência humana também poderia ser considerado. Embora tenha sido constatada a retirada de orquídeas epifíticas (restos de raízes no ritidoma), a freqüência de espécies ornamentais dos gêneros Cattleya e Brassavola sugere um bom estádio de conservação da mata estudada.

O índice de Shannon tem sido amplamente empregado para expressar diversidade arbórea de florestas brasileiras (Silva \& Leitão Filho, 1982; Cavassan et al., 1984; Jarenkow \& Baptista, 1987; Waechter \& Jarenkow, 1998), porém uma comparação direta com o valor acima não é possível, devido às diferenças de amostragem e de estimativas de participação quantitativa. Em dois inventários semelhantes, realizados em florestas brejosas do Rio Grande do Sul, Waechter (1992) obteve valores iguais a 2,886 e 4,049, respectivamente para uma área do litoral sul (Taim) e outra do litoral norte (Torres).

\section{AGRADECIMENTOS}

Ao Sr. Marino Pereira, pela permissão de trabalhar em sua propriedade. Aos colegas Lúcia Rebello Dillenburg e Luís Mauro Rosa, pela colaboração nas atividades de campo. Ao arquiteto Luís Fernando da Luz pela arte final das figuras (área de estudo e diagrama climático). Ao colega João André Jarenkow, pela leitura crítica e valiosas sugestões. 


\section{REFERÊNCIAS BIBLIOGRÁFICAS}

Araújo, D.S.D. 1987. Restingas: síntese dos conhecimentos para a costa sul e sudeste brasileira. Simp. Ecos. Costa Sul e Sudeste Brasil., 1, Cananéia, Brasil. v.1, p.333-347.

Araújo, D.S.D. 1992. Vegetation types of sandy coastal plains of tropical Brazil: a first approximation. In: Seeliger, U. Coastal plant communities of Latin America. San Diego, Academic. p.337-347.

Baptista, L.R.M., Ceroni, Z.S.V., Irgang, B.E., Longhi, H.M., Waechter, J.L., Miotto, S.T.S., Mariath, J.E.A., Rosito, J.M., Prado, J.F. \& Zanin, D. 1979. Levantamento florístico preliminar da Reserva Biológica do Lami, Porto Alegre, RGS. Porto Alegre, UFRGS. 30p. (NIDECO sér. Urbana no. 1).

Bennett, B.C. 1986. Patchiness, diversity, and abundance relationships of vascular epiphytes. Selbyana, 9(1):70-75.

Benzing, D.H. 1989. Vascular epiphytism in America. In: Lieth, H. \& Werger, M.J.A. Tropical rain forest ecosystems. Amsterdam, Elsevier. p.133-154. (Ecosystems of the world 14B).

Benzing, D.H. 1990. Vascular epiphytes. Cambridge, Cambridge University Press. 354p.

Braga, P.I.S. 1977. Aspectos biológicos das Orchidaceae de uma campina da Amazônia Central. Acta Amaz., 7(2):1-89. (Suplemento).

Brown, A.D. 1990. El epifitismo en las selvas montanas del Parque Nacional "El Rey", Argentina: composición florística y patrón de distribución. Rev. Biol. Trop., 38(2A): 155-166.

Catling, P.M. \& Lefkovitch, L.P. 1989. Associations of vascular epiphytes in a Guatemalan cloud forest. Biotropica, 21(1):35-40.

Cavassan, O., Cesar, O. \& Martins, F.R. 1984. Fitossociologia da vegetação arbórea da Reserva Estadual de Bauru, Estado de São Paulo. Rev. Brasil. Bot., 7(2):91-106.

Cottan, G. \& Curtis, J.T. 1956. The use of distance measures in phytosociological sampling. Ecology, 37(3):451-460. 
Dillenburg, L.R., Waechter, J.L., \& Porto, M.L. 1992. Species composition and structure of a sandy coastal plain forest in northern Rio Grande do Sul, Brazil. In: Seeliger, U. Coastal plant communities of Latin America. San Diego, Academic. p.349-366.

Evangelista, P.H.L. 1996. Levantamento florístico das pteridófitas epífitas da Reserva Volta Velha, Itapoá - SC. Curitiba, UFPR. 190p. (Dissertação de Mestrado).

Fontoura, T. 1995. Distribution patterns of five Bromeliaceae genera in Atlantic rainforest, Rio de Janeiro state, Brazil. Selbyana, 16(1):79-93.

Garcia-Franco, J.G. 1996. Distribución de epífitas vasculares en matorrales costeros de Veracruz, México. Acta Bot. Mexicana, 37:1-9.

Gentry, A.H. \& Dodson, C.H. 1987. Diversity and biogeography of neotropical vascular epiphytes. Ann. Missouri Bot. Gard., 74(2):205-233.

Henriques, R.P.B., Araújo, D.S.D. \& Hay, J.D. 1986. Descrição e classificação dos tipos de vegetação da restinga de Carapebus, Rio de Janeiro. Rev. Brasil. Bot., 9(2):173-189.

Hertel, R.J.G. 1959. Esboço fito-ecológico do litoral centro do Estado do Paraná. Forma et Functio, 1(6):47-78.

Hueck, K. 1955. Plantas e formação organogênica das dunas do litoral paulista. São Paulo, Secretaria da Agricultura. 130p.

Jarenkow, J.A. \& Baptista, L.R.M. 1987. Composição florística e estrutura da mata com araucária na Estação Ecológica de Aracuri, Esmeralda, Rio Grande do Sul. Napaea, (3):9-18.

Johansson, D.R. 1974. Ecology of vascular epiphytes in West African rain forests. Acta Phytogeogr. Suec., 59:1-129.

Kelly, D.L. 1985. Epiphytes and climbers of a Jamaican rain forest: vertical distribution, life forms and life histories. J. Biogeogr., 12:223-241.

Lemos, R.C., Azolim, M.A.D., Abrão, P.U.R. \& Santos, M.C.L. 1973. Levantamento de reconhecimento dos solos do estado do Rio Grande do Sul. Recife, Ministério da Agricultura. 431p.

Lindeman, J.C., Baptista, L.R.M., Irgang, B.E., Porto, M.L., Girardi-Deiro, A.M. \& Lorscheitter-Baptista, M.L. 1975. Estudos botânicos no Parque Estadual de Torres, Rio Grande do Sul - Brasil. II. Levantamento florístico da Planície do Curtume, da área de Itapeva e da área colonizada. Iheringia, sér. bot., (21):15-52.

Rev. Ciência e Natura, Santa Maria, 20: 43 - 66 , 1998. 
Lindman, C.A.M. 1906. A vegetação no Rio Grande do Sul. Porto Alegre, Livraria Universal. 356p.

Lüttge, U. 1997. Physiological ecology of tropical plants. Berlin, Springer. $384 p$.

Magurran, A.E. 1988. Ecological diversity and its measurement. London \& Sydney, Croom Helm. 179p.

Malme, G.O.A.N. 1936. Zur Kenntnis der Phanerogamenflora des Sandgebietes im Süden von Rio Grande do Sul. Svensk. Bot. Tidsk., 30(1):1-29.

Migenis, L.E. \& Ackerman, J.D. 1993. Orchid-phorophyte relationships in a forest watershed in Puerto Rico. J. Trop. Ecol., 9:231-240.

Moreno, J.A. 1961. Clima do Rio Grande do Sul. Porto Alegre, Secretaria da Agricultura, 42p.

Mota, F.S. 1951. Estudos do clima do estado do Rio Grande do Sul, segundo o sistema de W. Koppen. Rev. Brasil. Geogr., 13(2):275-284.

Mueller-Dombois, D. \& Ellenberg, H. 1974. Aims and methods of vegetation ecology. New York, John Wiley. 547p.

Nadkarni, N.M. 1985. An ecological overview and checklist of vascular epiphytes in the Monteverde cloud forest reserve, Costa Rica. Brenesia, (24):55-62.

Nimer, E. 1979. Climatologia do Brasil. Rio de Janeiro, IBGE-SUPREN. 421p.

Nunes, V.F. \& Waechter, J.L. 1998. Florística e aspectos fitogeográficos de Orchidaceae epifíticas de um morro granítico subtropical. Pesquisas, Bot., (48):157-191.

Pittendrigh, C.S. 1948. The bromeliad-anopheles-malaria complex in Trinidad I. The bromeliad flora. Evolution, 2(1):58-89.

Rambo, B. 1954. História da flora do litoral riograndense. Sellowia, 6(6):113172.

Rambo, B. 1961. Migration routes of the South Brazilian rain forest. Pesquisas, bot., 5(12):1-54.

Reitz, R. 1961. Vegetação da zona marítima de Santa Catarina. Sellowia, 13 (13): 17-115.

56 Rev. Ciência e Natura, Santa Maria, 20: 43 - 66 , 1998. 
Richards, P.W. 1952. The tropical rain forest. Cambridge, At the University Press. 450p.

Sanford, W.W. 1968. Distribution of epiphytic orchids in semi-deciduous tropical forest in Southern Nigeria. J. Ecol., 56(3):697-705.

Schimper, A.F.W. 1888. Die epiphytische Vegetation Amerikas. Jena, Gustav Fischer. 162p.

Senna, R.M. \& Waechter, J.L. 1997. Pteridófitas de uma floresta com araucária 1. Formas biológicas e padrões de distribuição geográfica. Iheringia, Sér. Bot., Porto Alegre (48):41-58.

Silva, A.F. \& Leitão-Filho, H.F. 1982. Composição florística e estrutura de um trecho da mata atlântica de encosta no município de Ubatuba (São Paulo, Brasil). Rev. Brasil. Bot., 5(1/2):43-52.

Sota, E.R. 1971. El epifitismo y las pteridófitas en Costa Rica (America Central). Nova Hedwigia, 21(2/4):401-465.

Sota, E.R. 1972. Las pteridófitas y el epifitismo en el Departamento del Choco (Colombia). An. Soc. Cient. Argent., 194(5/6):245-278.

Steege, H. \& Cornelissen, J.H.C. 1989. Distribution and ecology of vascular epiphytes in lowland rain forest of Guyana. Biotropica, 21(4):331-339.

Sugden, A.M. 1981. Aspects of the ecology of vascular epiphytes in two Colombian cloud forests. II. Habitat preferences of Bromeliaceae in the Serrania de Macuira. Selbyana, 5(3/4):264-273.

Sugden, A.M. \& Robins, R.J. 1979. Aspects of the ecology of vascular epiphytes in two Colombian cloud forests. I. The distribution of the epiphytic flora. Biotropica, 11(3): 173-188.

Ule, E. 1901. Die Vegetation von Cabo Frio an der Küste von Brasilien. Jahrb. Syst., 28:511-528.

Valdivia, P.E. 1977. Estudio botánico y ecológico de la región del Rio Uxpanapa, Vera Cruz, no. 4. Las epífitas. Biotica, 2(1):55-81.

Vareschi, V. 1980. Vegetationsokologie der Tropen. Stuttgart, Eugen Ulmer. $293 p$.

Veloso, H.P. 1952. O problema ecológico vegetação-bromeliáceas-anofelinos I. A presença relativa das formas aquáticas do A. (Kerteszia) spp. como índice de positividade das espécies de bromeliáceas. An Bot. Herb. Barbosa Rodrigues, 4(4):187-228. 
Veloso, H.P. \& Calabria, P.V. 1953. O problema ecológico vegetação bromeliáceas - anofelinos II. Avaliação quantitativa dos criadouros e das formas aquáticas dos Anofelinos do subgênero Kerteszia nos principais tipos de vegetação do município de Brusque, estado de Santa Catarina. An Bot. Herb. Barbosa Rodrigues, 5(5):7-35.

Waechter, J.L. 1985. Aspectos ecológicos da vegetação de restinga no Rio Grande do Sul, Brasil. Comun. Mus. Ciênc. PUCRS, sér. bot., (33):49-68.

Waechter, J.L. 1986. Epífitos vasculares da mata paludosa do Faxinal, Torres, Rio Grande do Sul, Brasil. Iheringia, sér. bot., (34):39-49.

Waechter, J.L. 1990. Comunidades vegetais das restingas do Rio Grande do Sul. Simp. Ecos. Costa Sul e Sudeste Brasil., 2, Águas de Lindóia, Brasil. v.3, p.228-248.

Waechter, J.L. 1992. O epifitismo vascular na Planície Costeira do Rio Grande do Sul. São Carlos, UFSCar. 163p. (Tese de Doutorado).

Waechter, J.L. 1998. Epiphytic orchids in eastern subtropical South America. Proc. World Orchid Conference, 15, Rio de Janeiro, Brasil. p.332-341.

Waechter, J.L. \& Jarenkow, J.A. 1998. Composição e estrutura do componente arbóreo nas matas turfosas do Taim, Rio Grande do Sul. Biotemas, 11(1):45-69.

Walter, H. 1971. Ecology of tropical and subtropical vegetation. Edinburgh, Oliver \& Boyd. 539p.

Walter, H. 1986. Vegetação e zonas climáticas. São Paulo, Editora Pedagógica e Universitária (EPU). 325p.

Whittaker, R.H. 1972. Evolution and measurement of species diversity. Taxon, 21(2/3): 213-251.

Zimmerman, J.K. \& Olmsted, Y.C. 1992. Host tree utilization by vascular epiphytes in a seasonally inundated forest (tintal) in Mexico. Biotropica, 24:402-407. 
Tabela 1. Lista das espécies de epífitos vasculares amostrados na restinga arbórea de Emboaba, Osório (RS), com indicação das espécies apenas observadas $\left({ }^{*}\right)$.

\section{ARACEAE}

Anthurium scandens (Aubl.) Engl.

Philodendron bipinnatifidum Schott ex Endl. $\left({ }^{\star}\right)$

BROMELIACEAE

Aechmea recurvata (KI.) L. B. Smith

Billbergia zebrina (Herb.) Lindl. ( $\left.{ }^{*}\right)$

Bromelia antiacantha Bertol. ( ${ }^{*}$ )

Tillandsia aëranthos (Loisel.) L. B. Smith

Tillandsia gardneri Lindl.

Tillandsia geminiflora Brongn.

Tillandsia stricta Soland.

Tillandsia usneoides (L.) L.

Vriesea friburgensis Mez

Vriesea gigantea Gaud. $\left(^{*}\right)$

Vriesea philippocoburgii Wawra $\left(^{*}\right)$

Vriesea platzmannii E. Morr.

Vriesea vagans (L. B. Smith) L. B. Smith (*)

CACTACEAE

Cereus hildmannianus K. Schum. ( $\left.{ }^{*}\right)$

Lepismium cruciforme (Vell.) Miq.

Rhipsalis teres (Vell.) Steud.

CECROPIACEAE

Coussapoa microcarpa (Schott) Rizz. $\left(^{*}\right)$

COMMELINACEAE

Tradescantia fluminensis Vell.

DRYOPTERIDACEAE

Rumohra adiantiformis (Forst.) Ching

MORACEAE

Ficus organensis (Miq.) Miq. ( ${ }^{\star}$ )

OPHIOGLOSSACEAE

Ophioglossum palmatum L. ( $\left.{ }^{*}\right)$ 
Tabela 1. Conclusão.

\section{ORCHIDACEAE}

Brassavola tuberculata Hook.

Campylocentrum aromaticum B. Rodr.

Campylocentrum linearifolium Schltr. \& Mansf.

Cattleya intermedia Grah. ex Hook.

Cattleya tigrina A. Rich.

Encyclia vespa (Vell.) Dressl.

Epidendrum pseudodifforme Hoehne \& Schltr.

Epidendrum rigidum Jacq. ( $\left.{ }^{*}\right)$

Gomesa crispa (Lindl.) KI. \& Rchb. f. $\left(^{*}\right)$

Lankesterella ceracifolia (B. Rodr.) Mansf.

Lophiaris pumila (Lindl.) Braem

Octomeria crassifolia Lindl. $\left({ }^{*}\right)$

Oncidium ciliatum Lindl.

Oncidium flexuosum Sims

Oncidium micropogon Rchb. f. (*)

Pleurothallis aquinoi Schltr. $\left(^{*}\right)$

Pleurothallis glumacea Lindl.

PIPERACEAE

Peperomia catharinae Miq.

Peperomia caulibarbis Miq.

Peperomia pereskiifolia (Jacq.) HBK.

\section{POLYPODIACEAE}

Campyloneurum nitidum (Kaulf.) Presl (*)

Microgramma squamulosa (Kaulf.) Sota $\left({ }^{\star}\right)$

Microgramma vacciniifolia (Langsd. \& Fisch.) Copel.

Pecluma sicca (Lindm.) Price $\left(^{*}\right)$

Polypodium catharinae Langsd. \& Fisch. $\left(^{*}\right)$

Polypodium hirsutissimum Raddi (*)

Polypodium meniscifolium Langsd. \& Fisch.

Polypodium pleopeltifolium Raddi

Polypodium squalidum Vell. $\left(^{\star}\right)$

\section{VITTARIACEAE}

Vittaria lineata (L.) J. E. Smith (*) 
Tabela 2. Espécies de forófitos amostrados na restinga arbórea de Emboaba, Osório (RS), com valores numéricos indicativos de densidade, tamanho e riqueza de epífitos vasculares.

\begin{tabular}{lrrrr}
\hline Espécie de Forófito & ni & $\begin{array}{r}\text { Dap } \\
\text { max }\end{array}$ & $\begin{array}{r}\text { Alt } \\
\text { max }\end{array}$ & $\begin{array}{r}\text { Sev } \\
\text { max }\end{array}$ \\
\hline Allophylus edulis (St.-Hil.) Radlk. & 2 & 12 & 8,0 & 6 \\
Butia capitata (Mart.) Becc. & 1 & 25 & 6,0 & 5 \\
Casearia sylvestris Sw. & 9 & 31 & 8,5 & 15 \\
Cereus hildmannianus K. Schum. & 2 & 33 & 6,0 & 11 \\
Chrysophyllum marginatum (Hook. \& Arn.) Radlk & 3 & 24 & 7,5 & 16 \\
Erythroxylum argentinum Schultz & 2 & 27 & 7,0 & 11 \\
Eugenia uniflora L. & 4 & 28 & 6,0 & 16 \\
Myrrhinium atropurpureum Schott & 1 & 14 & 5,0 & 10 \\
Myrsine umbellata Mart. ex A. DC. & 9 & 24 & 9,0 & 9 \\
Sebastiania commersoniana (Baill.) Smith \& Downs & 21 & 48 & 11,0 & 16 \\
Sideroxylon obtusifolium (Roem. \& Schult.) Penn. & 4 & 90 & 12,5 & 12 \\
Zanthoxylum hyemale St.-Hil. & 2 & 12 & 8,0 & 6 \\
\hline
\end{tabular}

$\mathrm{ni}=$ número de indivíduos amostrados (ni); $\max =$ valores máximos amostrados;

Dap = diâmetro à altura do peito $(\mathrm{cm}) ;$ Alt = altura total do forófito $(\mathrm{m}) ;$

Sev = número de espécies epifíticas vasculares sobre um único forófito amostrado. 
Tabela 3. Estimativas de freqüência e importância das espécies epifíticas vasculares amostradas na restinga arbórea de Emboaba, Osório (RS).

\begin{tabular}{|c|c|c|c|c|c|c|c|}
\hline ESPÉCIE EPIFÍTICA & $\mathrm{Nfi}$ & $\mathrm{FAi}$ & $\mathrm{FRi}$ & Sfi & $\mathrm{FAj}$ & FRj & Vie \\
\hline 01) Microgramma vacciniifolia & 55 & 91,7 & 9,3 & 12 & 100,0 & 6,7 & 8,01 \\
\hline 02) Tillandsia aëranthos & 57 & 95,0 & 9,6 & 11 & 91,7 & 6,1 & 7,89 \\
\hline 03) Aechmea recurvata & 43 & 71,7 & 7,3 & 11 & 91,7 & 6,1 & 6,71 \\
\hline 04) Vriesea friburgensis & 45 & 75,0 & 7,6 & 10 & 83,3 & 5,6 & 6,60 \\
\hline 05) Tillandsia usneoides & 43 & 71,7 & 7,3 & 10 & 83,3 & 5,6 & 6,43 \\
\hline 06) Tillandsia geminiflora & 39 & 65,0 & 6,6 & 10 & 83,3 & 5,6 & 6,09 \\
\hline 07) Rhipsalis teres & 36 & 60,0 & 6,1 & 10 & 83,3 & 5,6 & 5,84 \\
\hline 08) Peperomia catharinae & 31 & 51,7 & 5,2 & 10 & 83,3 & 5,6 & 5,42 \\
\hline 09) Oncidium ciliatum & 33 & 55,0 & 5,6 & 8 & 66,7 & 4,5 & 5,03 \\
\hline 10) Peperomia pereskiifolia & 26 & 43,3 & 4,4 & 9 & 75,0 & 5,0 & 4,71 \\
\hline 11) Lepismium cruciforme & 29 & 48,3 & 4,9 & 8 & 66,7 & 4,5 & 4,69 \\
\hline 12) Lophiaris pumila & 28 & 46,7 & 4,7 & 8 & 66,7 & 4,5 & 4,60 \\
\hline 13) Peperomia caulibarbis & 24 & 40,0 & 4,1 & 8 & 66,7 & 4,5 & 4,27 \\
\hline 14) Lankesterella ceracifolia & 22 & 36,7 & 3,7 & 7 & 58,3 & 3,9 & 3,82 \\
\hline 15) Tillandsia stricta & 13 & 21,7 & 2,2 & 8 & 66,7 & 4,5 & 3,33 \\
\hline 16) Polypodium pleopeltifolium & 8 & 13,3 & 1,4 & 5 & 41,7 & 2,8 & 2,07 \\
\hline 17) Cattleya intermedia & 8 & 13,3 & 1,4 & 4 & 33,3 & 2,2 & 1,79 \\
\hline 18) Brassavola tuberculata & 7 & 11,7 & 1,2 & 4 & 33,3 & 2,2 & 1,71 \\
\hline 19) Campylocentrum aromaticum & 10 & 16,7 & 1,7 & 3 & 25,0 & 1,7 & 1,68 \\
\hline 20) Tillandsia gardneri & 6 & 10,0 & 1,0 & 4 & 33,3 & 2,2 & 1,62 \\
\hline 21) Campylocentrum linearifolium & 6 & 10,0 & 1,0 & 3 & 25,0 & 1,7 & 1,35 \\
\hline 22) Cattleya tigrina & 4 & 6,7 & 0,7 & 3 & 25,0 & 1,7 & 1,18 \\
\hline 23) Encyclia vespa & 4 & 6,7 & 0,7 & 3 & 25,0 & 1,7 & 1,18 \\
\hline 24) Anthurium scandens & 3 & 5,0 & 0,5 & 2 & 16,7 & 1,1 & 0,81 \\
\hline 25) Polypodium meniscifolium & 2 & 3,3 & 0,3 & 2 & 16,7 & 1,1 & 0,73 \\
\hline 26) Tradescantia fluminensis & 3 & 5,0 & 0,5 & 1 & 8,3 & 0,6 & 0,53 \\
\hline 27) Vriesea platzmannii & 2 & 3,3 & 0,3 & 1 & 8,3 & 0,6 & 0,45 \\
\hline 28) Epidendrum pseudodifforme & 1 & 1,7 & 0,2 & 1 & 8,3 & 0,6 & 0,36 \\
\hline 29) Oncidium flexuosum & 1 & 1,7 & 0,2 & 1 & 8,3 & 0,6 & 0,36 \\
\hline 30) Pleurothallis glumacea & 1 & 1,7 & 0,2 & 1 & 8,3 & 0,6 & 0,36 \\
\hline 31) Rumohra adiantiformis & 1 & 1,7 & 0,2 & 1 & 8,3 & 0,6 & 0,36 \\
\hline
\end{tabular}

$\mathrm{Nfi}=$ número de forófitos individuais ocupados pela espécie $i$ epifítica;

$\mathrm{FAi}, \mathrm{FRi}$ = freqüência absoluta e relativa sobre forófitos individuais;

$\mathrm{Sfi}=$ número de forófitos específicos ocupados pela espécie $i$ epifítica;

$\mathrm{FAj}, \mathrm{FRj}$ = freqüência absoluta e relativa sobre forófitos específicos;

$\mathrm{Vle}=$ valor de importância epifítica $(F R i+F R j)$. 


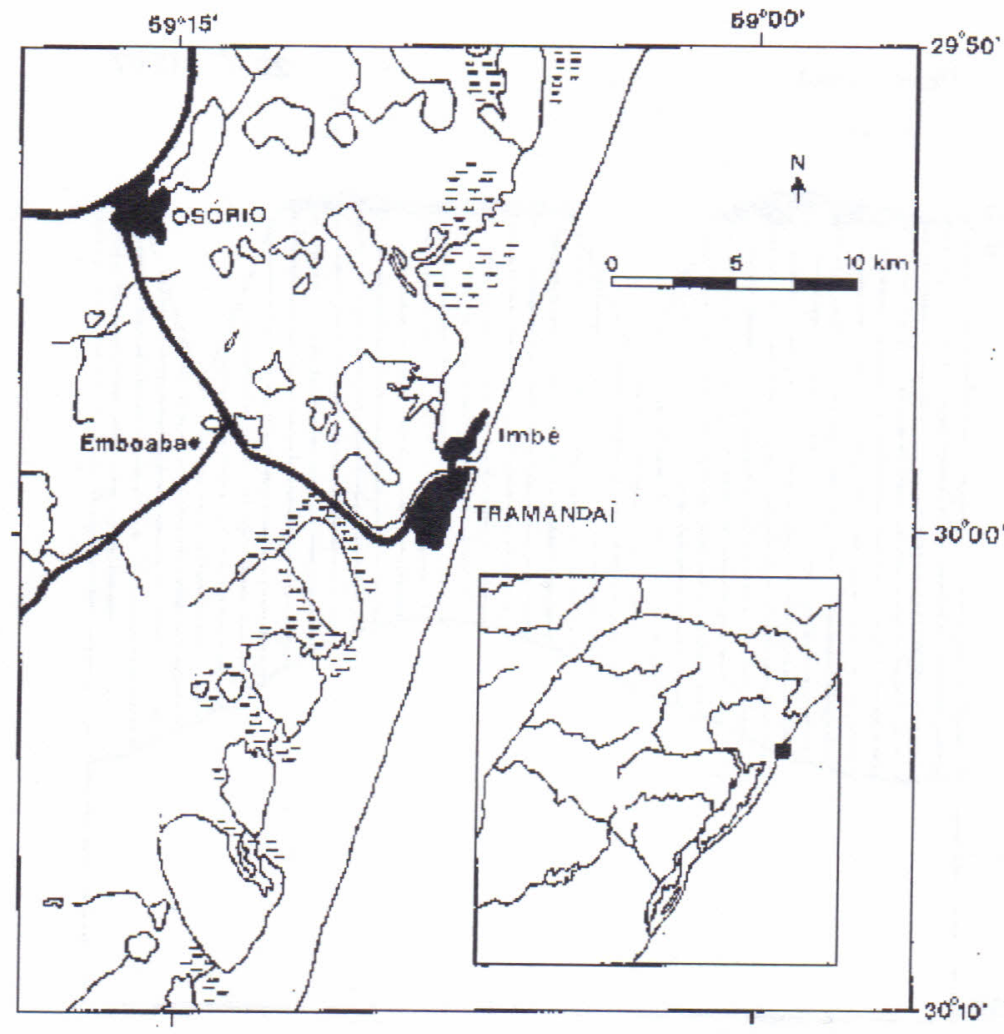

Figura 1. Localização da área de estudo (Emboaba), entre as sedes dos municípios de Osório, Imbé e Tramandai (RS). 


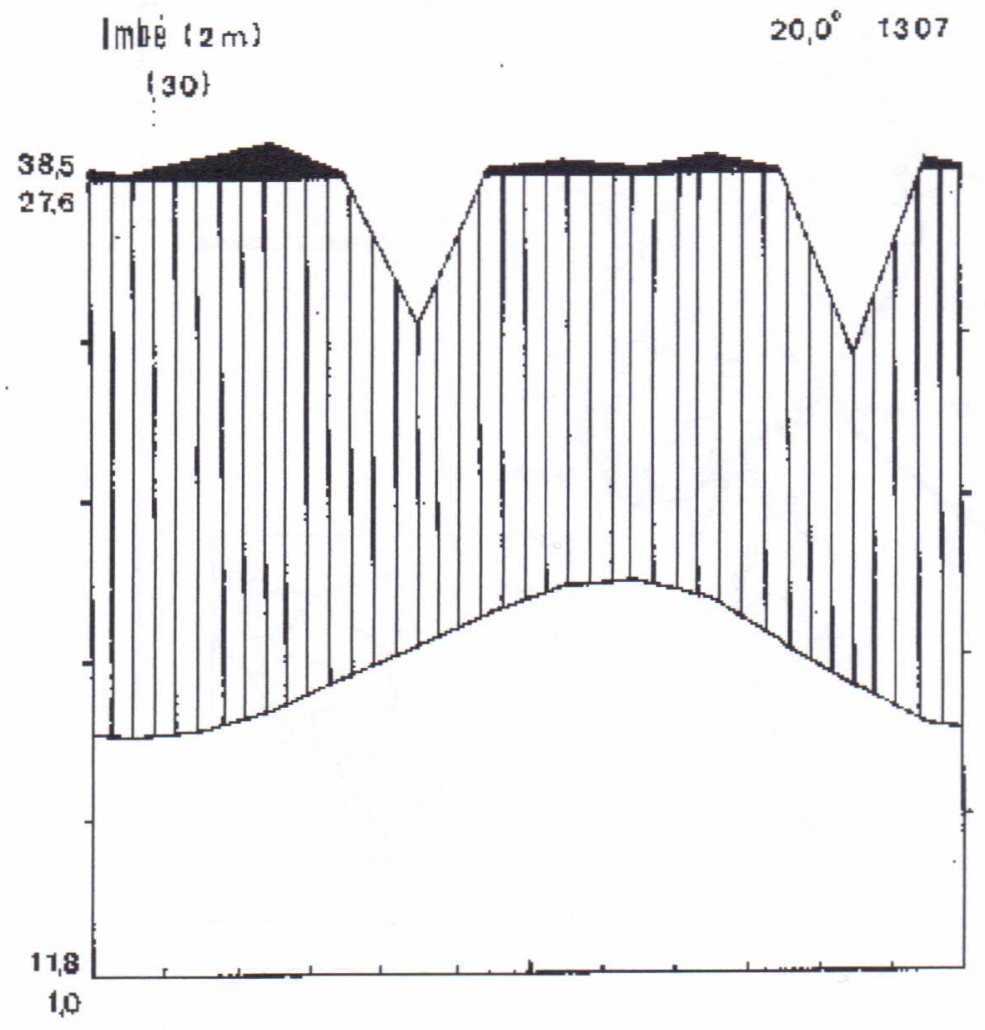

Figura 2. Diagrama climático da Estação Meteorológica de Imbé (2958'S, 5007’W, 2msm): as medidas de temperatura e pluviosidade correspondem, respectivamente, aos períodos de 1952-1987 e 1950-1985. 


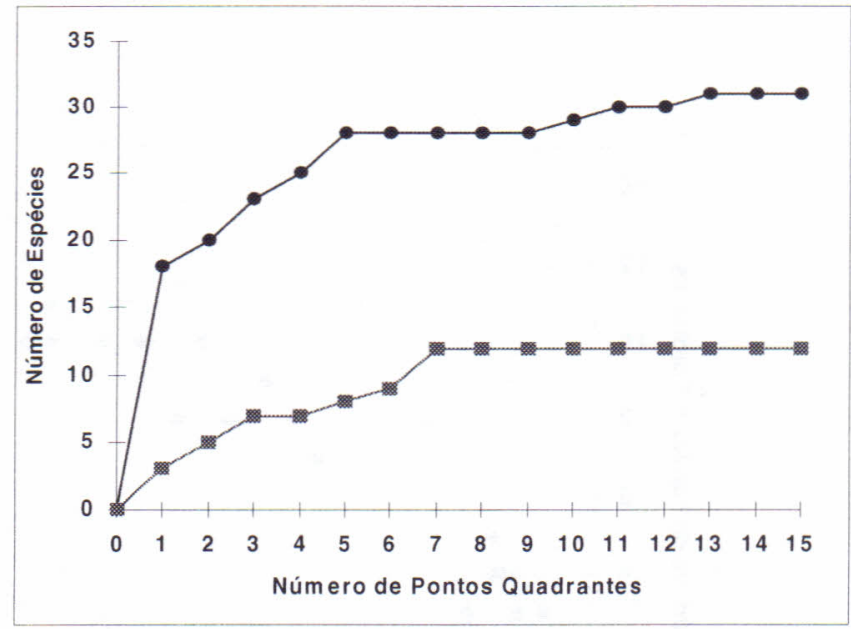

Figura 3. Relação cumulativa entre o número de pontos de amostragem e o número de espécies de forófitos (quadrados) e o número de espécies de epífitos vasculares (círculos), na mata de restinga de Emboaba (RS).

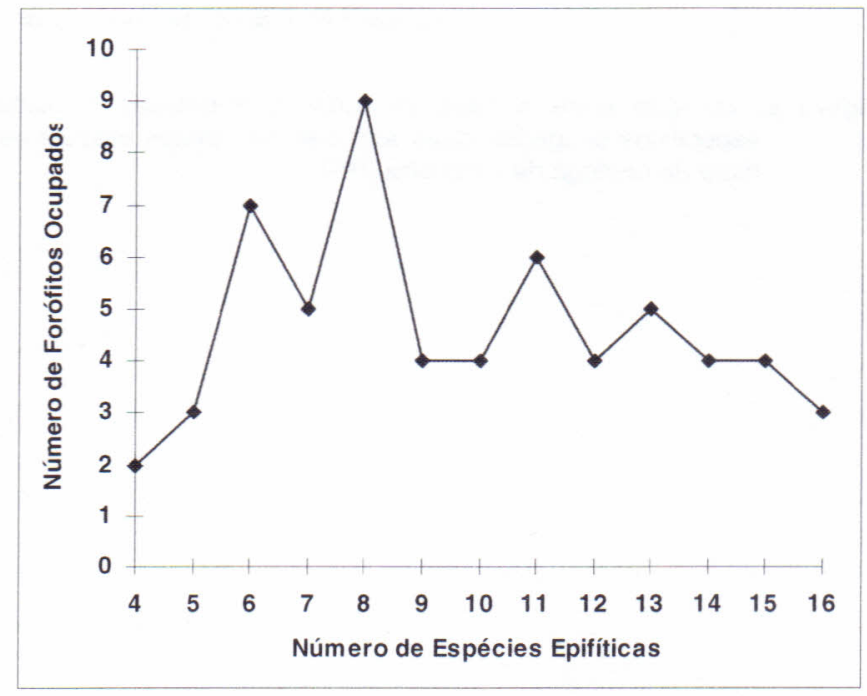

Figura 4. Distribuição freqüencial da riqueza epifítica vascular sobre forófitos individuais, na mata de restinga de Emboaba (RS). O valor modal máximo (8) situa-se mais próximo da riqueza mínima (4) do que da riqueza máxima (16). 


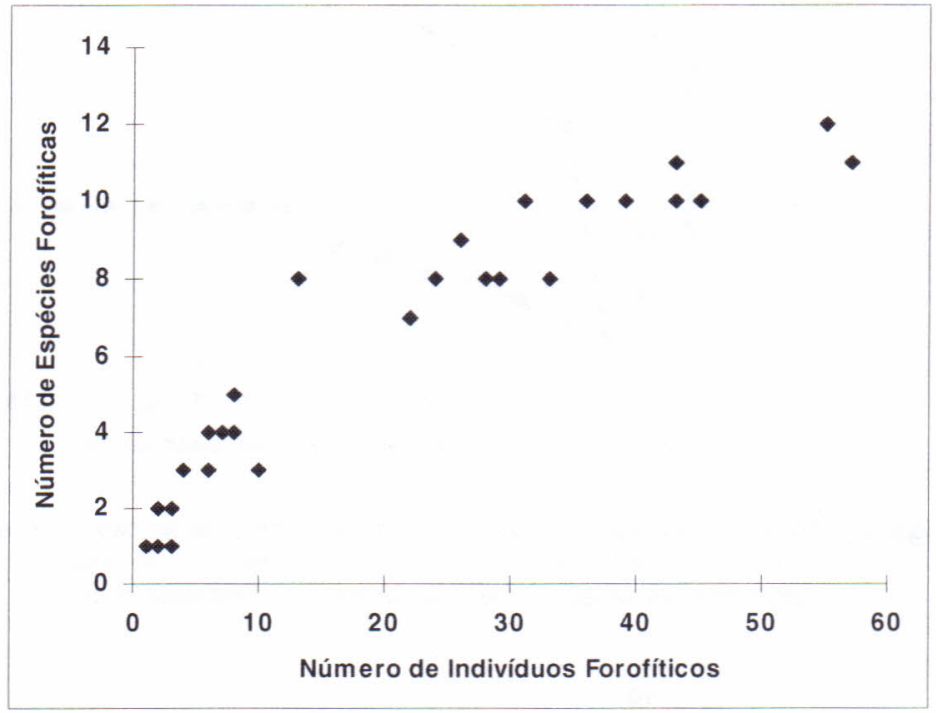

Figura 5. Relação entre número de forófitos individuais e número de forófitos específicos ocupados pelas espécies de epífitos vasculares amostradas na mata de restinga de Emboaba (RS). 